\title{
Effect of Different Membership Functions on Fuzzy Power System Stabilizer for Synchronous Machine Connected to Infinite Bus
}

\author{
Slimane Touil \\ Department of Electrical \\ Engineering, El-oued \\ University, Algeria
}

\author{
Djilani Ben Attous \\ Department of Electrical \\ Engineering, El-oued \\ University, Algeria
}

\section{ABSTRACT}

The effectiveness of power system stabilizer (PSS) in providing damping and improving the dynamic response is well established. In this paper focuses, the performance of single machine infinite bus is studied with fuzzy power system stabilizer (FPSS). The generator is represented by the standard K-coefficients as second order system. The mechanism to discuss the rules is described and the performance is investigated for trapezoidal, triangular and Gaussian membership functions of input and output variables. For FPSS, speed deviation and acceleration deviation are taken as input. The system is simulated in SIMULINK environment and performance is investigated for transients due to change in input of varying magnitude.

\section{Keywords}

Power System Stabilizer, Synchronous Machine, Single Machine Infinite Bus System, Fuzzy Logic, Simulink

\section{INTRODUCTION}

The conventional power plants of 1950s were equipped with continuously acting automatic voltage regulators to improve the transient stability of system. The high gain of these voltage regulators has a destabilizing effect on power system. The power oscillations of small magnitude and high frequency, which often persisted in power system, present the limitation to the amount of power transmitted within the system. Improved performance has been achieved by adding damping to the system by employing power system stabilizers (PSS). The conventional PSS, which uses lead-lag compensation, is resulting into poor performance under different loading conditions [1]-[2] as the gain settings are designed for specific condition. In the early years, a stabilizer providing good performance under various operating conditions could not be designed. The fuzzy logic control has been suggested as a possible solution to this problem. Metwally and Malik [2] described fuzzy power system stabilizer using generator speed and output power variation as the controller input variables. Taliyat et al. [3] presented the design of augmented fuzzy power system stabilizer to enhance power system stability. Hiyama [4] derived acceleration, speed deviation and phase deviation of generator from measured real power signal. Majid et al. [5] presented a fuzzy controller in which speed deviation and rotor acceleration were taken as inputs. Juan et al. [6] compared the fuzzy and rule based power system stabilizers. Gupta et al. [7]. proposed a robust FPSS using speed deviation and rotor acceleration as inputs for multi machine system and studied the performance using different defuzzification methods.

Moodley et al. [8] investigated the effect of FLPSS in a multimachine environment. Ferreira et al. [9] proposed a FPSS including a fuzzy PI controller to improve steady state behavior of a system. Lim [10] proposed a fuzzy logic control scheme using only one input signal which is derived as the linear combination of signal, signal derivative and signal integral.

In this paper, the performance of single machine infinite bus system with fuzzy power system stabilizer is presented. The generator is represented by second order system with the help of standard K-constant representation. The speed deviation and acceleration deviations are taken as the inputs

Performance is studied for trapezoidal, gaussian and triangular membership functions of fuzzy sets and compared with conventional lead-lag compensator. The simulation has been implemented in SIMULINK

\section{SYSTEM MODELING}

The system comprises of synchronous machine, excitation system and power system stabilizer model. The system model is developed as follows:-

\subsection{Synchronous Machine Model}

The configuration of synchronous machine connected to infinite bus through transmission network is represented as the Thevenin's equivalent shown in Fig. 1

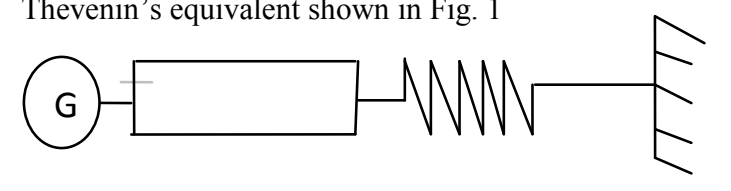

Generator transmission line Infinite bus

Fig.1: The equivalent of synchronous machine connected to infinite bus

The equations governing the machine model [11] are:-

$$
S \Delta \omega_{r}=\frac{1}{2 H}\left(T_{m}-T_{e}-K_{D} \Delta \omega_{r}\right)
$$


$S \Delta \delta=\omega_{\circ} \Delta \omega_{r}$

Where,

$\Delta T e=K_{1} \Delta \delta+K_{2} \Delta \psi_{f d}$

$\Delta \psi_{f d}=\frac{K_{3}}{1+T_{3} s}\left(\Delta E_{f d}-K_{4} \Delta \delta\right)$

Where, $T_{m}$ and $T_{e}$ are the prime mover input and electrical output torques respectively, $\mathrm{H}$ is the inertia constant, $\delta$ and $\omega$ are rotor angle and speed respectively

\subsection{Excitation System Model}

The thyristor excitation system as shown in Fig. 2 is considered. The nonlinearity associated with the ceilings EFMAX and EFMIN, is ignored for small-disturbance studies.

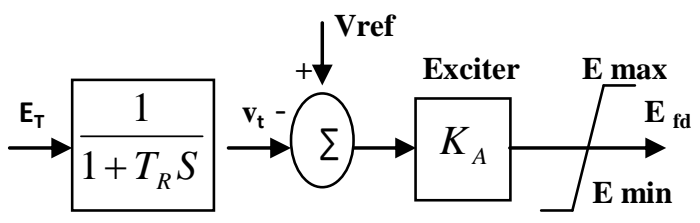

Fig. 2: Thyristor excitation system

The equations governing the exciter model are:-

$$
\begin{aligned}
& s \Delta v_{1}=\frac{1}{T_{R}}\left(\Delta E_{t}-\Delta v_{1}\right) \\
& E_{f d}=k_{A}\left(V_{r e f}-v_{1}\right) \\
& \Delta E_{t}=K_{5} \Delta \delta+K_{6} \Delta \psi_{f d}
\end{aligned}
$$

\subsection{Power System Stabilizer Model}

The PSS is used to provide damping to electromechanical oscillations. The PSS counters the oscillations by forcing the change in excitation level appropriately. Without PSS, the reduced damping in power system is due to phase lags resulted by the field time constants and the phase lags in the normal voltage regulation loop. The PSS uses phase compensation by adjusting the timing of correction signal opposing the rotor oscillations.

A power system stabilizer can Therefore increase the generator's damping coefficient. The PSS as shown in Fig. 3 has three components, the phase compensation block, the signal washout block and gain block. The phase compensation block provides the appropriate phase lead characteristics to compensate for the phase lag between exciter input and generator electrical torque. The signal washout block serves as high pass filter, with time constant $T_{W}$ high enough to allow signals associated with oscillations in $\omega_{r}$ to pass unchanged. The stabilizer gain KSTAB determines the amount of damping introduced by PSS. For the conventional PSS following transfer functions is considered.

$$
\begin{aligned}
& \Delta v_{2}=\frac{s T_{W}}{1+s T_{W}}\left(K_{S T A B} \Delta \omega_{r}\right) \\
& \Delta v_{s}=\frac{1+s T_{1}}{1+s T_{2}}\left(\Delta v_{2}\right)
\end{aligned}
$$

$T_{W}$ is the washout filter time constant. The implementation of the PSS in K constant model is shown in Fig. 4.

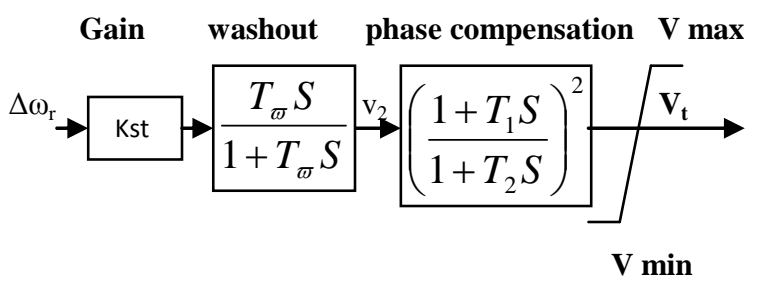

Fig. 3: Conventional lead-lag PSS

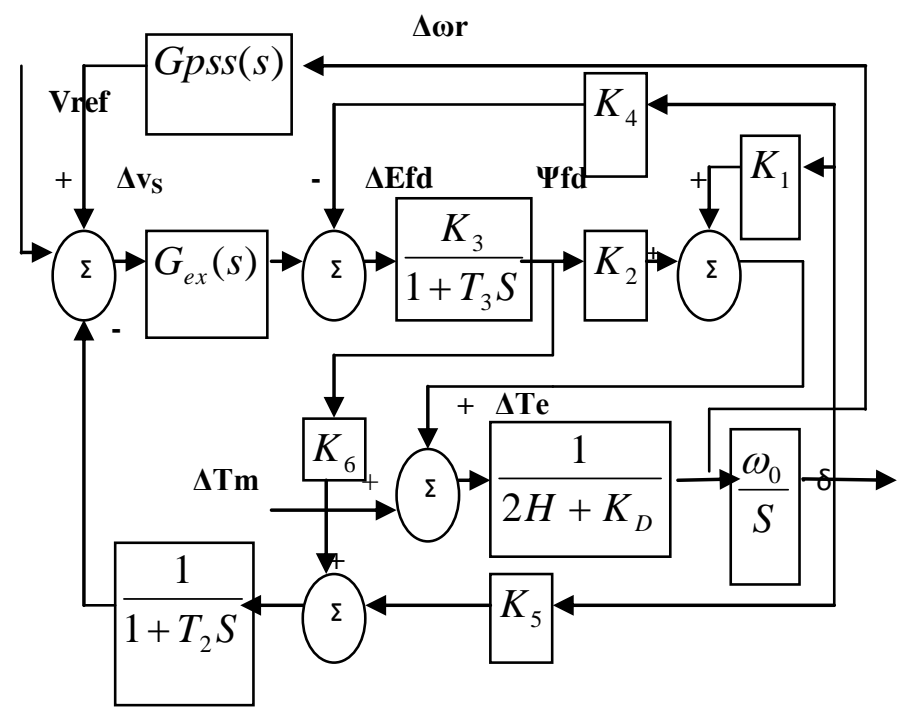

Fig. 4: Block diagram representation with AVR and PSS

\section{FUZZY CONTROLLED POWER SYSTEM STABILIZER}

\subsection{Fuzzy Control System}

Since the concept of fuzzy logic given by Zadeh in 1965, it has found applications in various areas including a controller for power system stabilizer [12]. A fuzzy controller, as shown in Fig. 5, comprises of four stages: fuzzification, a nowledge base, decision making and defuzzification. The fuzzification interface converts input data into suitable linguistic values that can be viewed as label fuzzy sets. The knowledge base comprises knowledge of application domain and attendant control goals by means of set of linguistic control rules. The decision making is the aggregation of output of various control rules that simulate the capability of human decision 
making. The defuzzification inference performs scale mapping, which converts the range of values of output variables into corresponding universe of discourse

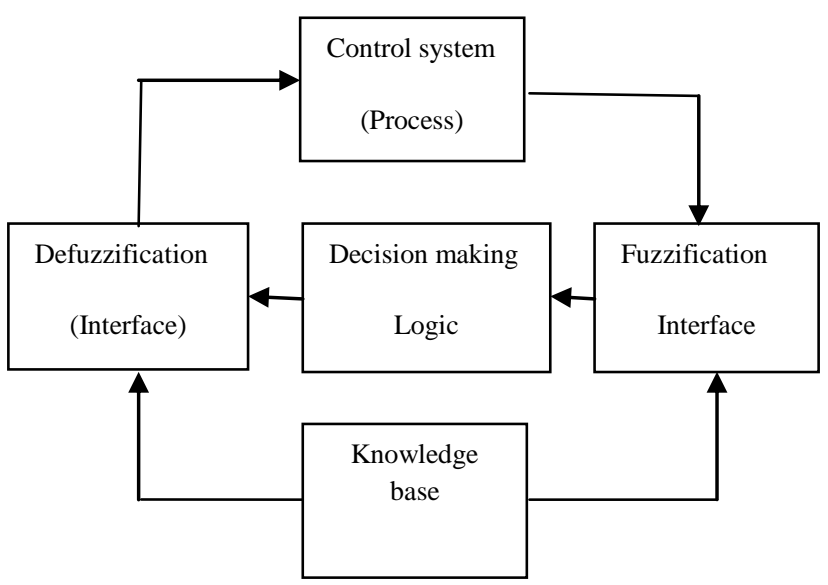

Fig. 5: The principle design of fuzzy logic controller

The fuzzy controller design consists of the following steps:-

1) Identification of input and output variables.

2) Construction of control rules.

3) Establishing the approach for describing system state in

terms of fuzzy sets, i.e. establishing fuzzification method and fuzzy membership functions. Selection of the compositional rule of inference. Defuzzification method transformation of the fuzzy control statement into specific control actions.

\subsection{Generating Membership Functions}

The method proposed by Hong et al. [13] which helps in generating membership functions has been used in FPSS. This method partitions a set of data into classes that can be used to derive membership functions. The method for obtaining membership function for a variable is explained as:-

Step1. Given a data set, there are $n$ training samples. The values for the parameter x $\vec{X}=x_{1}, x_{2} \ldots x_{n}$,

are sorted into ascending order, denoted as

$\vec{Y}=\operatorname{Sort}[\vec{X}]=y_{1}, y_{2} \ldots y_{n}$,

Step2. Calculate the difference between the adjacent data:

$\operatorname{diff}_{i}=y_{i+1}-y_{i}$, for $\mathrm{i}=(1,2,3, \ldots \mathrm{n}-1)$,

Step3. The similarities between adjacent values are found and mapped into real numbers between 0 and 1 as:-

$s_{i}=\left\{\begin{array}{l}\left\{1-\frac{\text { diff }_{i}}{C * \sigma_{s}} \text { for.diff }_{i} \leq C * \sigma_{s}\right\} \\ 0 . \text { otherwise }\end{array}\right\}$ $\sigma_{s} s$ is the standard deviation of diff $_{i}$ and

$\mathrm{C}$ is the control parameter used to determine the shape of the membership function

Step4. The data is grouped according to similarities. A

Threshold value $\alpha$ divides adjacent values into classes. If the similarity is greater than the threshold value, then the two adjacent values belong to the same class, otherwise the values are divided into different classes by creating a new class

if $\left(s_{i} \geq \alpha\right)$ then.. $y_{i}, y_{i+1} \in C_{i}$

Else. $y_{i} \in C_{i}, y_{i+1} \in C_{i+1}$

where $C_{i}$ and $\mathrm{Ci}+1$ denote two distinct classes for the same parameter.

Step5. The triangular membership function for each class is defined. For class $\mathrm{j}$, it consists of three points: the central vertex point, $b_{j}$, and the two endpoints, $a_{j}$ and $c_{j}$. The central vertex point is determined for each class and is calculated by the given formula:

$b_{j}=\frac{y_{i} * s_{i}+y_{i+1} * \frac{s_{i}+s_{i+1}}{2}+. . y_{k-1} * \frac{s_{k-2}+s_{k-1}}{2}+y_{k} * s_{k-1}}{s_{i}+\frac{s_{i}+s_{i+1}}{2}+. . \frac{s_{k-2}+s_{k-1}}{2}+s_{k-1}}$

Where, $k$ represents the ending data index for this class, i.e., data $y_{i}$ through $y_{k}$ fall into class $\mathrm{j}$, and $s_{i}$ is the similarity between $y_{i}$ and $y_{i+1}$. The endpoints $a_{j}$ and $c_{j}$, are obtained using interpolation as:-

$a_{j}=b_{j}-\frac{b_{j}-y_{i}}{1-\mu_{j}\left(y_{i}\right)}, c_{j}=b_{j}+\frac{y_{k}-b_{j}}{1-\mu_{j}\left(y_{k}\right)}$

$\mu_{j}\left(y_{i}\right)$ and $\mu_{j}\left(y_{k}\right)$ is the membership expressed as:-

$\mu_{j}\left(y_{i}\right)=\mu_{j}\left(y_{k}\right)=\min \left(s_{i}, s_{i+1}, \ldots . s_{k-1}\right)$

\subsection{Fuzzy Based PSS}

The fuzzy power system stabilizer a two-input, a singleoutput component. The two inputs are change in angular speed and rate of change of angular speed whereas output of fuzzy logic controller is a voltage signal. The membership function of a variable is expressed into seven fuzzy sets defined in Table. 1 .

Where, 
Table.1: Fuzzy stets of variable

\begin{tabular}{|c|c|}
\hline NB & NEGATIVE BIG \\
\hline NM & NEGATIVE MEDIUM \\
\hline NS & NEGATIVE SMALL \\
\hline $\mathbf{Z E}$ & ZERO \\
\hline PS & POSITIVE SMALL \\
\hline PM & POSITIVE MEDIUM \\
\hline PB & POSITIVE BIG \\
\hline
\end{tabular}

The membership function maps the crisp values into fuzzy variables. The choice of membership functions has an important bearing on the performance of the fuzzy logic based PSS. A set of rules which define the relation between the inputs and output of fuzzy controller are defined using the linguistic variables. The knowledge required to generate the fuzzy rules can be derived from an offline simulation. However, it has been noticed that, for monotonic systems, a symmetrical rule table is very appropriate, although sometimes it Where, $k$ represents the maximum data index value within the class.

may need slight adjustment based on the behavior of the specific system. If the system dynamics are not known or are highly nonlinear, trial-and-error procedures and experience play an important role in defining the rules. The two inputs, speed and acceleration, result in 49 rules for each machine as shown in Table.2. The typical rules are having the following structure

Rule 1: If speed deviation is NM (negative medium) AND acceleration is PS (positive small) then voltage (output of fuzzy PSS) is NS (negative small).

Rule 2: If speed deviation is NB (negative big) AND acceleration is NB (negative big) then voltage (output of fuzzy PSS) is NB (negative big).Table.2: Rules for fuzzy PSS

\begin{tabular}{|l|l|l|l|l|l|l|l|}
\hline \multirow{2}{*}{$\begin{array}{l}\text { Speed } \\
\text { deviation }\end{array}$} & \multicolumn{6}{|c|}{ Acceleration } \\
\cline { 2 - 8 } & NB & NM & NS & ZE & PS & PM & PB \\
\hline NB & NB & NB & NB & NB & NM & NM & NS \\
\hline NM & NB & NM & NM & NM & NS & NS & ZE \\
\hline NS & NM & NM & NS & NS & ZE & ZE & PS \\
\hline ZE & NM & NS & NS & ZE & PS & PS & PM \\
\hline PS & NS & ZE & ZE & PS & PS & PM & PM \\
\hline PM & ZE & PS & PS & PM & PM & PM & PB \\
\hline PB & PS & PM & PM & PB & PB & PB & PB \\
\hline
\end{tabular}

After replacing the conventional PSS block by fuzzy controller block, the representation of fuzzy logic controller implemented on single machine infinite bus system can be shown in the Fig. 6 . The fuzzy module has two inputs namely the angular velocity and its derivative i.e. angular acceleration and output parameter as voltage. These are normalized by gains Kin1, Kin2 and Kout respectively to match the range on which the membership functions are defined. Parameters Kin1, Kin2 and Kout are tuned to give the desired response.

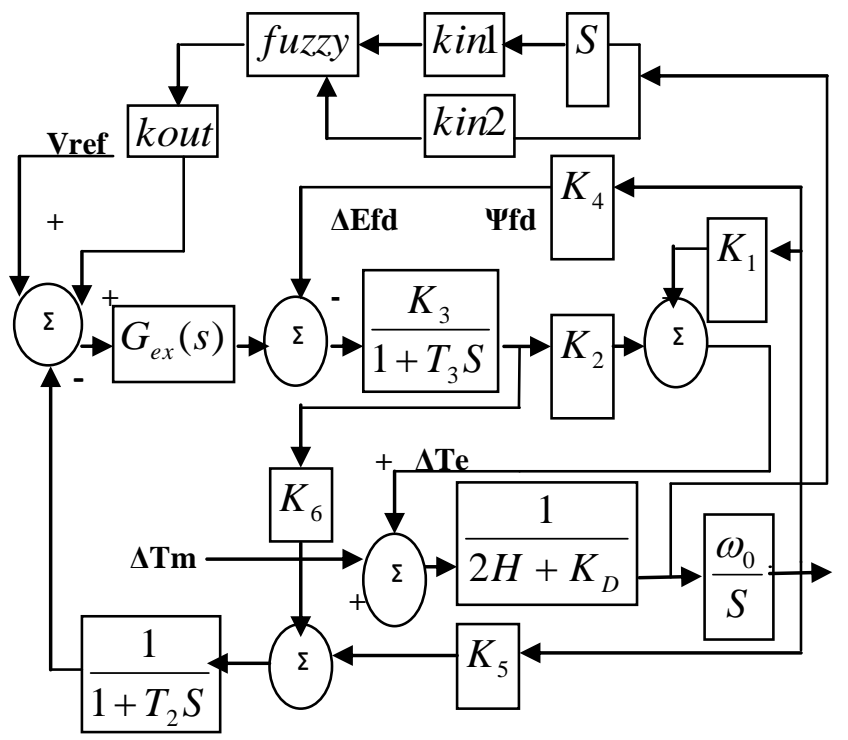

Fig. 6: Single machine infinite bus system with FPSS.

\section{RESULTS AND DISCUSSION}

The performance of single machine infinite bus system with lead-lag PSS and fuzzy PSS has been studied in SIMULINK environment. The fuzzy stabilizer has been Modeled in fis editor of matlab. The SIMULINK model of the system with fuzzy PSS is shown in Fig. 7. Corresponding to the system data specified in Appendix, the K-coefficients are calculated as $\mathrm{K} 1=0.7636, \mathrm{~K} 2=0.8644, \mathrm{~K} 3=0.3231, \mathrm{~K} 4=1.4189$, $\mathrm{K} 5=-$ $0.1463, \mathrm{~K} 6=0.4167$. The scaling factors associated with input and output variables are tuned and taken as Kin $1=1.8$, Kin2 $=29.58$, Kout $=1.05$. For such constants, which are in Fig. 7 , the performance has been studied under different conditions.

Although the stability problem in practical power system is mainly due to poor damping, the constant K5 has important bearing on system performance. Its value normally found to be negative for high reactance and high power output, a practical situation. The K5 can have positive value for low reactance and low power output. Therefore, the performance is studied for small 0.05 p.u. change in input for both $\mathrm{K} 5$ negative (-0.1463) and K5 positive (0.1463) and response characteristics are shown Fig. 8 and Fig. 9 respectively. 


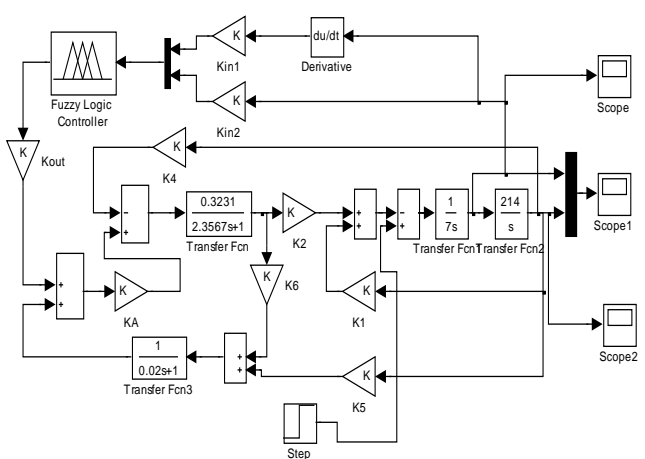

Fig. 7: SIMULINK model with fuzzy logic based PSS

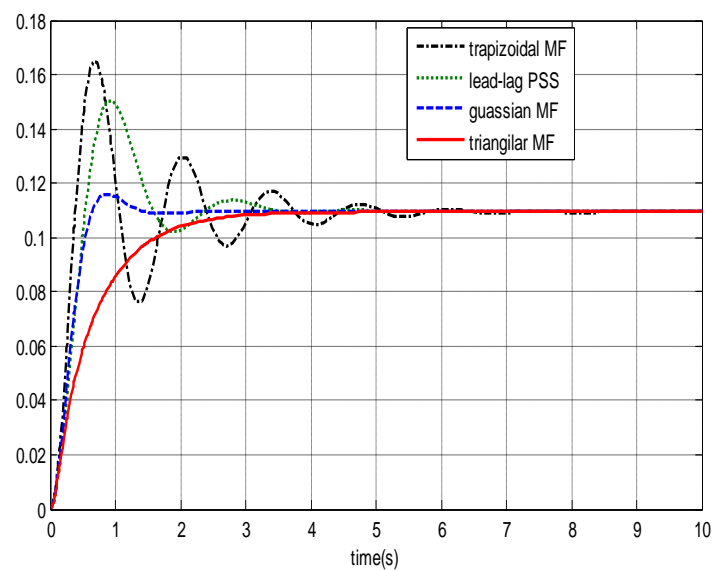

Fig. 8 (a): Angular position for a 0.05pu change in mechanical input with $\mathrm{K5}$ positive

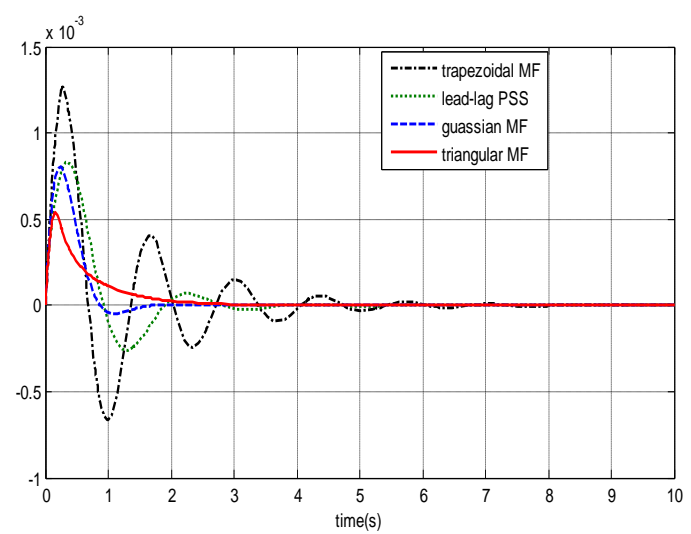

Fig. 8 (b): Angular speed for a 0.05pu change in mechanical input with $\mathrm{K} 5$ positive

As shown in Fig. 8(a) and Fig. 8(b), the oscillations are more pronounced in case of inputs and outputs having trapezoidal membership functions for $\mathrm{K} 5$ positive and the system become stable after a long time of 9 seconds approximately. With K5 negative, generally the practical case, the performance characteristics are shown in Fig. 9(a) and Fig. 9(b). The unstable behavior is resulted if the trapezoidal membership function is used with negative value of K5. As evident from Fig. 8 and Fig. 9, the performance with trapezoidal membership functions is unsatisfactory and thus this membership function is not suited for the PSS design. The performance of lead-lag PSS and PSS using Gaussian and triangular membership functions is comparable and the system becomes stable after in nearly 3 seconds

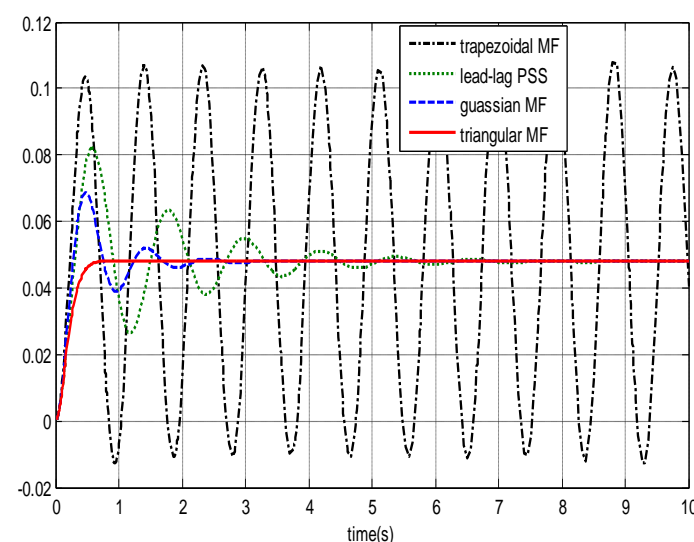

Fig. 9 (a): Angular position for a 0.05pu change in mechanical input with $\mathrm{K} 5$ negative.

With the Gaussian membership, the performance may be affected by the change in the spread of the membership function. The effect of change in the spread of Gaussian membership is therefore studied and the results are presented in Fig. 10 for negative value of K5. With the increase in the spread of Gaussian membership function, oscillations are reduced and system becomes stable after 3.5 seconds. However, the unstable response is resulted with decreased spread.

The oscillations are reduced considerably in case the inputs and outputs having triangular membership functions and system become stable after 0.5 seconds approximately.

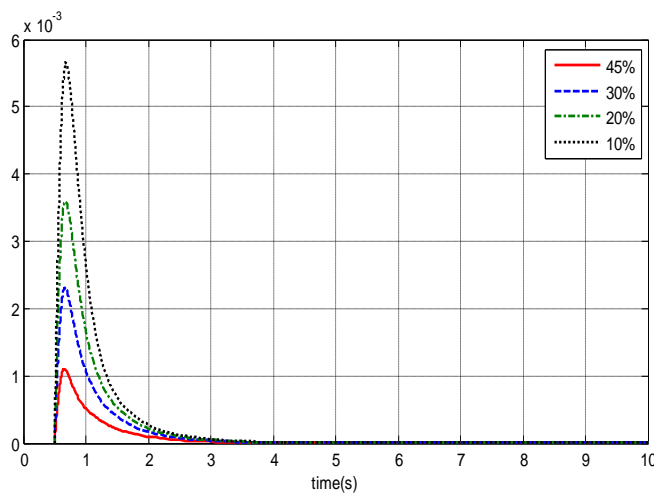

Fig. 9 (b): Angular speed for a 0.05pu change in mechanical input with $\mathrm{K5}$ negative. 


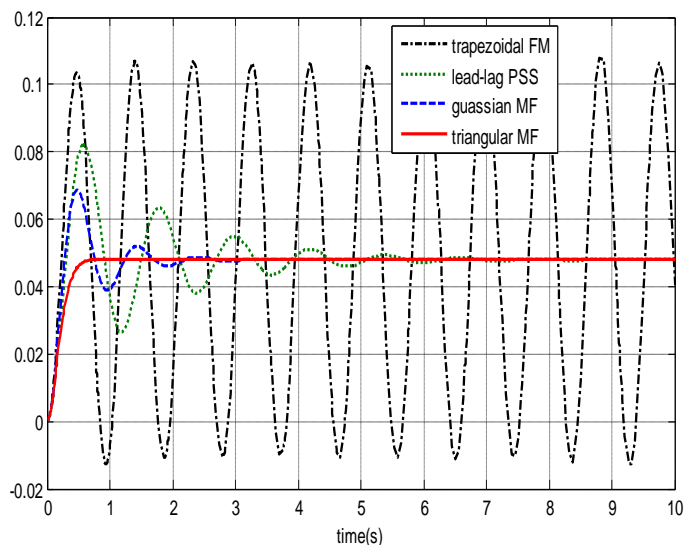

Fig. 10 (a): Angular position for a 0.05pu change in mechanical input with $\mathrm{K5}$ negative with the change in spread of Gaussian membership function

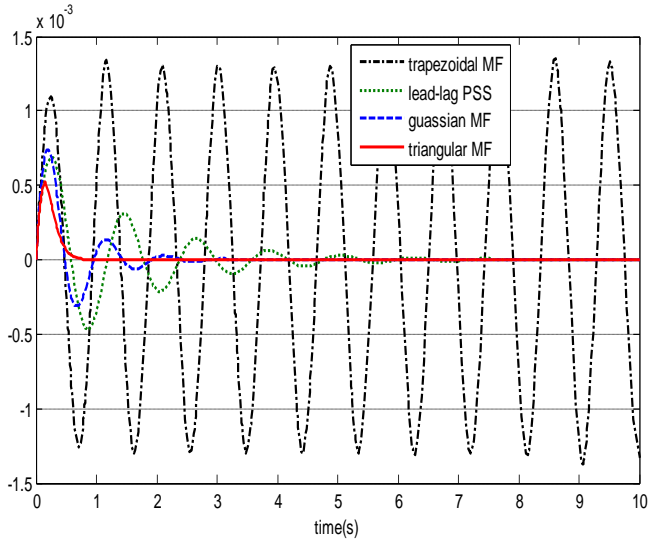

Fig. 10 (b): Angular position for a 0.05pu change in mechanical input with $\mathrm{K5}$ negative with the change in spread of Gaussian membership function.

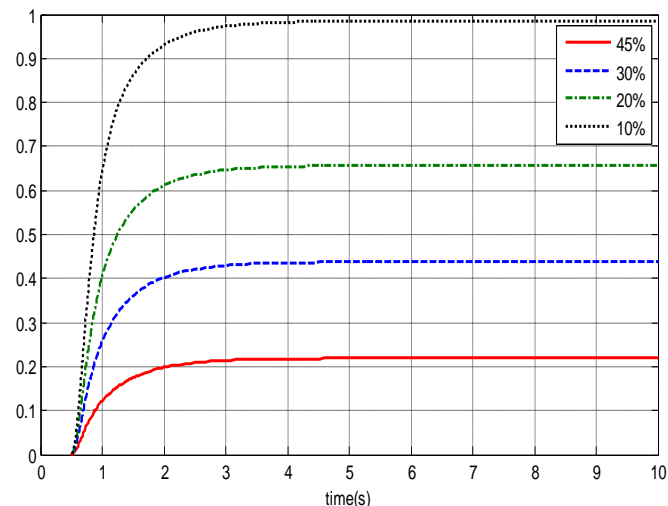

Fig. 11 (a): Angular position for different operating conditions using fuzzy PSS

The response for different operating conditions using triangular membership functions is investigated for negative value of $\mathrm{K} 5$ and the performance characteristics are shown in Fig. 11 for $10 \%, 20 \%, 30 \%$ and $45 \%$ change in mechanical input. With triangular membership functions, the response is coming out to the stable in all the cases with very less oscillations thus enhancing the stability of a system. As seen in Fig. 8-11, the performance of FPSS with triangular membership function is superior in comparison to the performance with other PSS

\section{CONCLUSION}

The simulation studies support that the fuzzy PSS provide the better damping of oscillations as compared to conventional PSS (lead-lag). However, the choice of Member ship functions has an important bearing on the damping of oscillations. The oscillations are more pronounced in case of trapezoidal membership functions. The response with gauss membership functions having increased spread is comparable to triangular membership functions. However, the performance of FPSS with triangular membership functions is superior compared to other PSS.

\section{APPENDIX}

Synchronous machine parameters:

$\mathrm{P}=0.9, \mathrm{Q}=0.3, \mathrm{Et}=1.0, \mathrm{f}=50, \mathrm{Xd}=1.81, \mathrm{Xq}=1.76, \mathrm{Xd} 1=0.3$, $\mathrm{XL}=0, \mathrm{Xe}=0.65, \mathrm{Ra}=0.003$, Tdo1=8.0, $\mathrm{H}=3.5, \omega=314, \mathrm{Kd}=0$, $\mathrm{TR}=0.02$, ETmag=1.0, Ladu=1.65, Ladu=1.60, Rfd=0.0006, Lfd $=0.153, \mathrm{Ksd}=0.849, \mathrm{Ksq}=0.849$, KsdI=0.434, KsqI=0.434, ASAT $=0.031$, BSAT $=6.93, \Psi 1=0.8$,

Frequency of oscillation(in $\mathrm{rad} / \mathrm{sec})=10$. The standard IEEE type ST1A excitation system model has been considered for the study with parameters as: $-\mathrm{KA}=200, \mathrm{TR}=0.02$. The parameters of PSS are:- $\mathrm{T} 1=0.154, \mathrm{~T} 2=0.033$.

\section{REFERENCES}

[1] F W Keay and W H south, "Design of Power System Stabilizer Sensing Frequency Deviation", IEEE Transactions on Power Apparatus and Systems, vol. 90, pp.707- 713, March 1971.

[2] K A El-Metwally and O P Malik, "Fuzzy Logic Based Power System Stabilizer", IEEE Proc- Gener.Transm. Distri., vol. 142, pp.277-281, May 1995.

[3] H Taliyat, J Sadeh and R Ghazi "Design of Augmented Fuzzy Logic Power System Stabilizer to Enhance Power System Stability" IEEE Transactions on Energy Conversion, vol 11, pp, 97-103, March 1996.

[4] T Hiyama, "Development of Fuzzy Logic Power System Stabilizer and Further Studies", IEEE International Conference on Systems, Man and Cybernetics '99, vol. 6 , pp.545-550.

[5] S Majid, H A Rahman and O B Jais, "Study of Fuzzy Logic Power System Stabilizer",IEEE Student onference on Research and Development 2002, pp. 335- 339.

[6] ] Jaun, L H Herron and A Kalam, "Comparison of Fuzzy Logic Based and Rule Based Power System Stabilizer", IEEE Conference on Control Application, pp.692- 697, sept.1992.

[7] R Gupta, D K Sambariya and R Gunjan, "Fuzzy Logic Based Robust Power System Stabilizer for a Multimachine Power System”,IEEE ICIT, pp. 1037-1042, Dec 2006.

[8] G V Moodley, G D Jennings, R G Harley and M T Wishart, "Fuzzy Logic Power System Stabilizer in Multi 
Machine Stability Studies" IEEE AFRICON, pp. 843848, Sept. 1996.

[9] A M D Ferreira, J A L Barreiros and J R Brito-de-Souza, "Fuzzy Power Pystem Stabilizer including a Fuzzy PI Controller", UKACC International Conference on Control, IEE, Sept 1998.

[10] C M Lim, "Application of a new Fuzzy Logic Control Scheme for Stability Enhancement-Simulation and Experimental Results", IEEE International Conference on Fuzzy Systems, vol.3, pp. 1339-1346, March 1995.

[11] T J Rose, "Fuzzy Logic With Engineering Applications", McGraw-Hill.Inc, Newyork, 1997

[12] T P Hong and C. Lee, "Induction of Fuzzy Rules and Membership Functions from Training Examples," Fuzzy Sets and Systems, vol. 84, pp. 33-47, Nov. 1996.

[13] P Kundur, "Power System Stability and Control", McGraw-Hill 1994.
[14] J H Chow and J J Sanchez-Gasca, "Pole Placement Designs of Power System Stabilizers", IEEE Transactions on Power Systems, vol. 4, pp. 271-277, Feb 1989.

[15] Y Y Hsu“Design of Proportional-integral Power System Stabilizer", IEEE Transactions on Power systems, vol. 1, pp. 46-52, May 1986.

[16] Y N Yu and Q H Li, "Pole Placement Power System Stabilizers Design of an Unstable Nine Machine System", IEEE Transactions on Power Systems , vol.5, pp. 353-358, May 1990.

[17] C J Wu and Y Y Hsu, "Design of Self-tuning PID Power System Stabilizer for Multimachine Power System", IEEE Transactions on Power Systems, vol. 3, pp. 10591064, Aug. 1998. 